\title{
Oligodendroglioma anaplásico quístico: Reporte de caso
}

\author{
Javier Illescas $\mathrm{C}^{1^{*}}$, Alejandro Vega $\mathrm{G}^{2}$, Carmen Amezcua $\mathrm{H}^{3}$, Erick Gómez $\mathrm{A}^{4}$, Laura Chávez $\mathbf{M}^{4}$.
}

1. Residente Alta Especialidad en Resonancia Magnética, Hospital General de México "Dr. Eduardo Liceaga". Facultad de Medicina, Universidad Nacional Autónoma de México Ciudad de México, México.

2. Jefe del Servicio de Radiología e Imagen Diagnostica, Radiólogo adscrito Departamento de Resonancia Magnética. Hospital General de México "Dr. Eduardo Liceaga". Facultad de Medicina, Universidad Nacional Autónoma de México. Ciudad de México, México.

3. Jefa del Servicio de Radiología e Imagen Diagnostica, Radiólogo adscrito Departamento de Resonancia Magnética, Hospital General de México "Dr. Eduardo Liceaga". Facultad de Medicina, Universidad Nacional Autónoma de México. Ciudad de México, México.

4. Departamento de Neuropatología, Servicio de Patológia, Hospital General de México "Dr. Eduardo Liceaga". Facultad de Medicina, Universidad Nacional Autónoma de México. Ciudad de México, México.

\section{Anaplastic cystic oligodendroglioma: Case report}

Resumen: Los oligodendrogliomas anaplásicos son gliomas infiltrantes grado III de la organización mundial de la salud (OMS). Son tumores poco frecuentes y representan el 5-10\% de todas las neoplasias intracraneales primarias. Su incidencia es de 0.3 por 100.000 habitantes por año en Estados Unidos. Con frecuencia se presentan en adultos entre los 40-60 años de edad. Los síntomas principales pueden ser déficit motor, déficit cognitivos y síntomas de aumento de la presión intracraneal. Su comportamiento en resonancia magnética muestra un aspecto heterogéneo con necrosis, degeneración quística y hemorragia intratumoral. Las presentaciones quísticas extensas son poco frecuentes. Reportamos el caso de un oligodendroglioma anaplásico de aspecto predominantemente quístico en una mujer joven.

Palabras clave: Aspecto quístico, Oligodendroglioma anaplásico, Resonancia magnética (RM).

Abstract: Anaplastic oligodendrogliomas are grade III infiltrating gliomas of the World Health Organization (WHO). They are rare tumors and represent $5-10 \%$ of all primary intracranial neoplasms. Its incidence is 0.3 per 100.000 inhabitants per year in the United States. They often occur in adults between 40-60 years of age. The main symptoms may be motor deficit, cognitive deficits and symptoms of increased intracranial pressure. Its behavior in MRI shows a heterogeneous appearance with necrosis, cystic degeneration and intratumoral hemorrhagic. Extensive cystic presentations are rare. We report the case of an anaplastic oligodendroglioma of predominantly cystic appearance in a young woman.

Keywords: Anaplastic oligodendroglioma, Cystic appearance, Magnetic resonance imaging (MRI).

Illescas J, et al. Oligodendroglioma anaplásico quístico: Reporte de caso. Rev Chil Radiol 2020; 26(1): 12-16. *Correo electrónico: Javier Illescas Cárdenas / xafeil@hotmail.com

Trabajo enviado el 03 enero de 2020. Aceptado para publicación el 20 de marzo de 2020.

Introducción

El oligodendrocito, como lo reconoció Robertson en 1900 , forma parte de la familia de células neurogliales y se encuentra predominantemente en la sustancia blanca del sistema nervioso central ${ }^{(1)}$.

Los oligodendrogliomas son tumores intracraneales que representan el $5-25 \%$ de todos los gliomas y el $5-10 \%$ de todas las neoplasias intracraneales primarias $^{(2)}$.

Con la actualización de la clasificación de la OMS de tumores del sistema nervioso central (SNC) en
2016, el diagnóstico de oligodendroglioma se realiza mediante la identificación de un glioma infiltrante difuso con mutación IDH y codeleción $1 \mathrm{p} 19 \mathrm{q}^{(2,3,4,5)}$.

Algunas veces los marcadores moleculares no están disponibles (en muchos países o centros regionales) o algunas veces son equívocos. En tales casos, el diagnóstico vuelve solo a las características histológicas y se denomina No especificado de otra manera (NOS) ${ }^{(3)}$.

La clasificación de la OMS de los tumores del SNC, por lo tanto, reconoce cuatro diagnósticos rela- 
cionados con oligodendrogliomas ${ }^{(6)}$ : oligodendroglioma (grado II de la OMS): IDH-mt, 1p19q co-eliminado, oligodendroglioma anaplásico (grado III de la OMS): IDH-mt, 1p19q co-eliminado, oligodendroglioma NOS (OMS grado II): IDH y 1p19q indeterminado, oligodendroglioma anaplásico NOS (OMS grado III): IDH y 1p19q indeterminado ${ }^{(3,6)}$.

La distinción entre oligodendroglioma (que es mutante IDH y codeletado $1 p / 19 q$ ) y el astrocitoma es clínicamente significativa. En el pasado, la mayoría de los gliomas grado II de la OMS y muchos gliomas grado III de la OMS fueron tratados con radioterapia sola, independientemente del subtipo, debido a la falta de beneficio comprobado para la quimioterapia. Más recientemente, se ha demostrado que la combinación de radioterapia seguida de quimioterapia con procarbazina, lomustina y vincristina (PCV) mejora significativamente la supervivencia en pacientes con gliomas de grado II y III de la OMS que albergan codeleción 1p / 19q (oligodendrogliomas en la definición de la OMS 2016) ${ }^{(7)}$.

\section{Caso clínico}

Mujer de 44 años, residente en la ciudad de México, ama de casa, sin antecedentes personales de interés. Presenta una historia de 3 meses de evolución con cefalea holocraneana opresiva de moderada a alta intensidad con hasta EVA 8/10, que se acompaña de nausea y vómito, de predominio matutino. No refiere factores exacerbantes, ni atenuantes; presenta crisis parciales motoras en brazo derecho (Jaksonianas). Ocasionalmente presentaba crisis convulsivas tónico clónicas generalizadas, disminución de fuerza en hemicuerpo derecho de manera gradual, se ha mantenido en tratamiento con oxcarbazepina 300 mg cada 12 horas y levetiracetam 1 g. cada 12 horas. Al examen físico neurológico se presenta con Glasgow de 15 puntos, orientada en tiempo, lugar y persona, funciones cognitivo corticales: lenguaje fluente, comprende, nómina y repite. Gnosias y praxias ideatorias e ideomotoras conservadas, memoria semántica, episódica, de trabajo y procedimental sin alteraciones. Tono y trofismo conservado, fuerza proximal y distal $4 / 5$ en hemicuerpo izquierdo, $3 / 5$ en hemicuerpo derecho.

La RM mostró una lesión intra-axial, fronto-parietal derecha sólido-quística, con un gran componente quístico, con sangrado intralesional, que presenta focos de restricción a la difusión y tras la administración de medio de contraste se evidencia refuerzo hacia la superficie dorsal (Figuras 1 y 2).

El examen histopatológico mostró en la Inmunohistoquímica Olig2 (400X), positivo nuclear en células neoplásicas. Inmunohistoquímica PAGF (400X), positivo citoplásmico en células neoplásicas. Inmunohistoquímica Ki67 (100X), positivo nuclear en el $20 \%$ de las células neoplásicas (Figura $3 \mathrm{H}, \mathrm{I}, \mathrm{J}$ ), con lo que se hace el diagnóstico de oligodendroglioma anaplásico NOS grado III de la OMS.

\section{Discusión}

En el registro de los Estados Unidos, la tasa de incidencia anual ajustada de oligodendroglioma se estima en 0.26 casos por cada 100.000 habitantes. Las tasas de incidencia de oligodendroglioma anaplásico se estiman en 0.11 casos por cada 100.000 habitantes.

Ocurren predominantemente en adultos, con un pico entre los 40 y 60 años de edad, los pacientes con tumores de bajo grado, son un poco más jóvenes que aquellos con tumores de alto grado (2). La relación hombre mujer 2:1(6).

Los oligodendrogliomas anaplásicos demuestran mayor densidad celular, mayor actividad mitótica, proliferación microvascular y necrosis ${ }^{(3,8)}$.

La RM es el método de elección para la evaluación de los oligodendrogliomas, con información morfológica detallada y actualmente aporta datos sobre el metabolismo y el flujo sanguíneo con técnicas avanzadas, como la espectroscopia y perfusión ${ }^{(9)}$. Su rol fundamental se basa en la evaluación diagnóstica; orientación quirúrgica y radioterapia; seguimiento y monitoreo del tratamiento(2).

Con mayor frecuencia los oligodendrogliomas grado II y III de la OMS ocurren en la corteza y sustancia blanca subcortical supratentorial, siendo el lóbulo frontal y el lóbulo temporal las ubicaciones más comunes ${ }^{(1,2)}$. Debido a su ubicación periférica, el adelgazamiento focal, la remodelación o incluso la erosión del cráneo suprayacente no es raro(4).

Se manifiestan típicamente como una lesión de morfología redonda u oval, generalmente hipointensa en comparación con la materia gris en las imágenes ponderadas en $\mathrm{T} 1$ e hiperintensa en comparación con la materia gris en imágenes potenciadas en T2, con una heterogeneidad comúnmente marcada. Existe calcificación gruesa en hasta el $90 \%$ de los casos. Una banda giriforme de mineralización cortical hace que el diagnóstico de oligodendroglioma sea altamente probable, ya que el oligodendroglioma es el tumor cerebral que más frecuentemente calcifica. Además, presenta escaso edema perilesional ${ }^{(1,2,8)}$.

Puede ocurrir degeneración quística, necrosis, hemorragia intratumoral, focos de restricción a la difusión y presencia de zonas de reforzamiento con el medio de contraste, tales características tienden a apuntar hacia un comportamiento anaplásico del tumor ${ }^{(2)}$. Al ser un tumor con red capilar intermedia, tienden a sangrar espontáneamente y esas áreas hemorrágicas se reabsorben y se cavitan. La presencia de la red capilar hace que los valores de volumen sanguineo relativo en estudios de perfusión no tengan una buena correlación con el grado histológico.

Los oligodendrogliomas anaplásicos pueden contener áreas de necrosis, incluida una necrosis en 


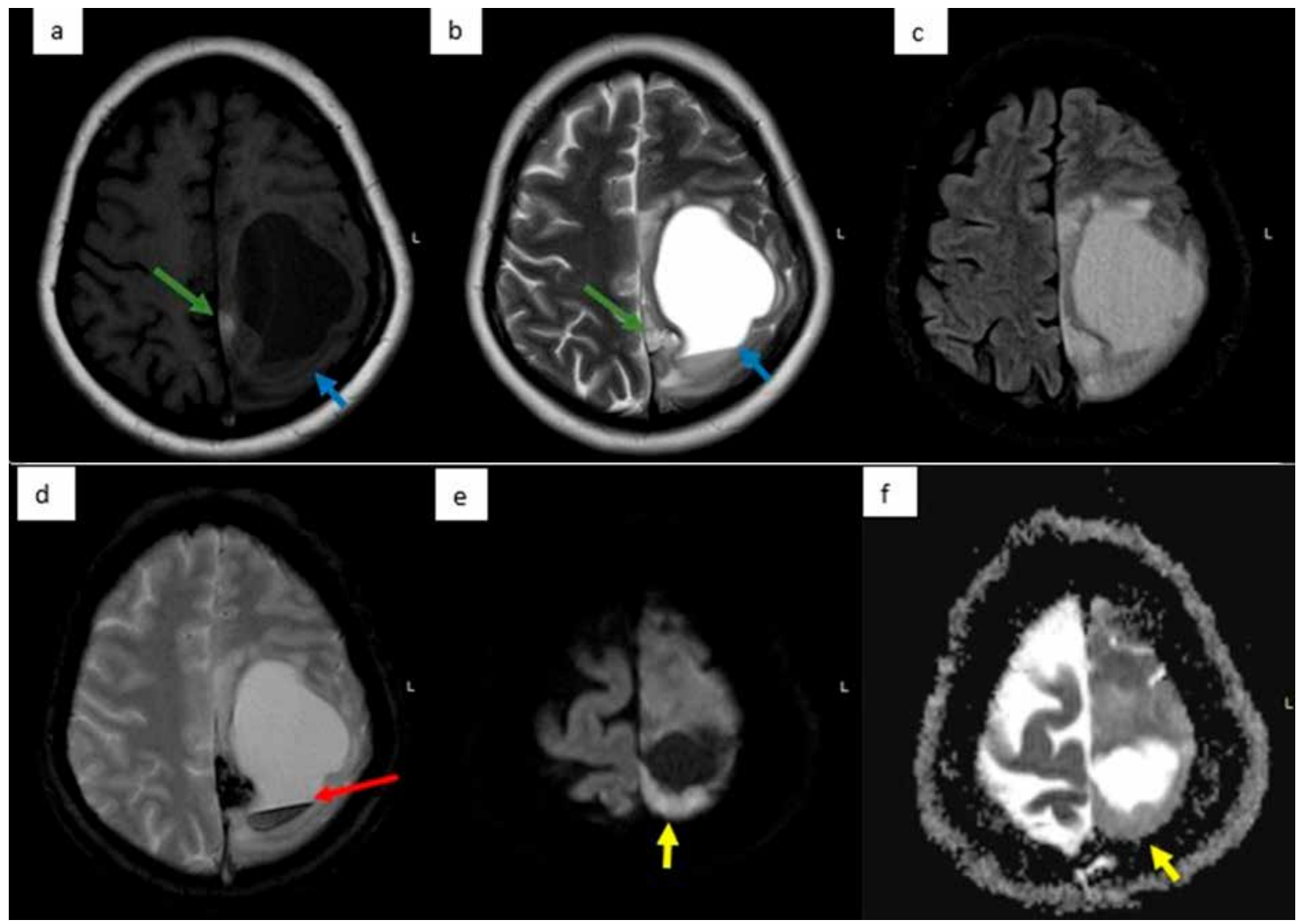

Figura 1a,b,c: Se observa lesión neoplásica parenquimatosa en localización fronto-parietal derecha, de contornos lobulados, bien definidos, aspecto quístico, en su interior con nivel líquido-líquido hacia la superficie caudal (flecha azul); la lesión muestra parte de su componente hacia la superficie interhemisférica que se comporta hiperintenso en T1, T2, y FLAIR en relación a sangrado (flecha verde), la neoplasia en conjunto con el edema perilesional condiciona efecto de volumen con obliteración de los espacios aracnoideos adyacentes. d: En secuencia eco de gradiente hemo se observa depósitos minerales en relación a depósitos de hemosiderina hacia la superficie intehemisférica, depósitos de hemosiderina en el interior de la lesión en la región caudal, formando el nivel líquido-líquido (flecha roja). e: en secuencia de difusión b1000 se identifica hacia la superficie más dorsal y caudal de la lesión, foco aislado de restricción en la región periférica que se corrobora con el mapa ADC imagen f: lo que indica zona de alta celularidad (flecha amarilla).

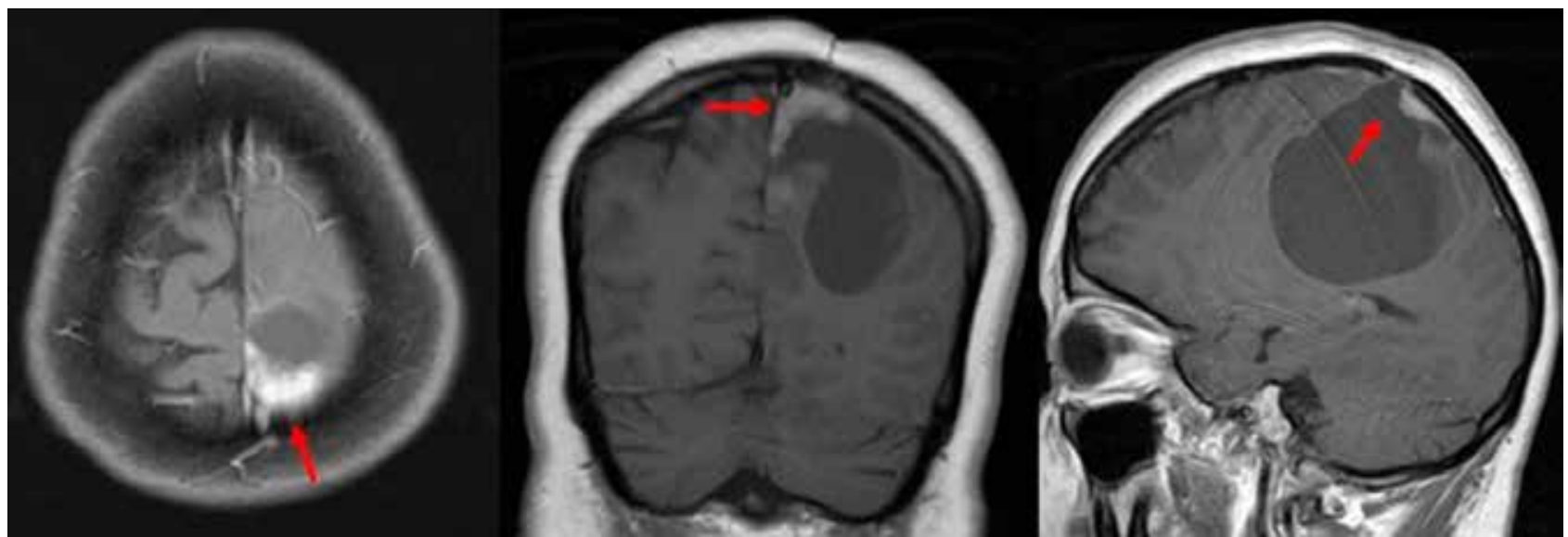

Figura 2: Tras la administración de medio de contraste intravenoso se evidencia reforzamiento en la región periférica de la lesión (flecha roja), hacia la superficie dorsal y caudal donde se identificaba focos de restricción en secuencia de difusión. Imágenes en planos coronal y sagital donde se ve la extensión de la lesión. 


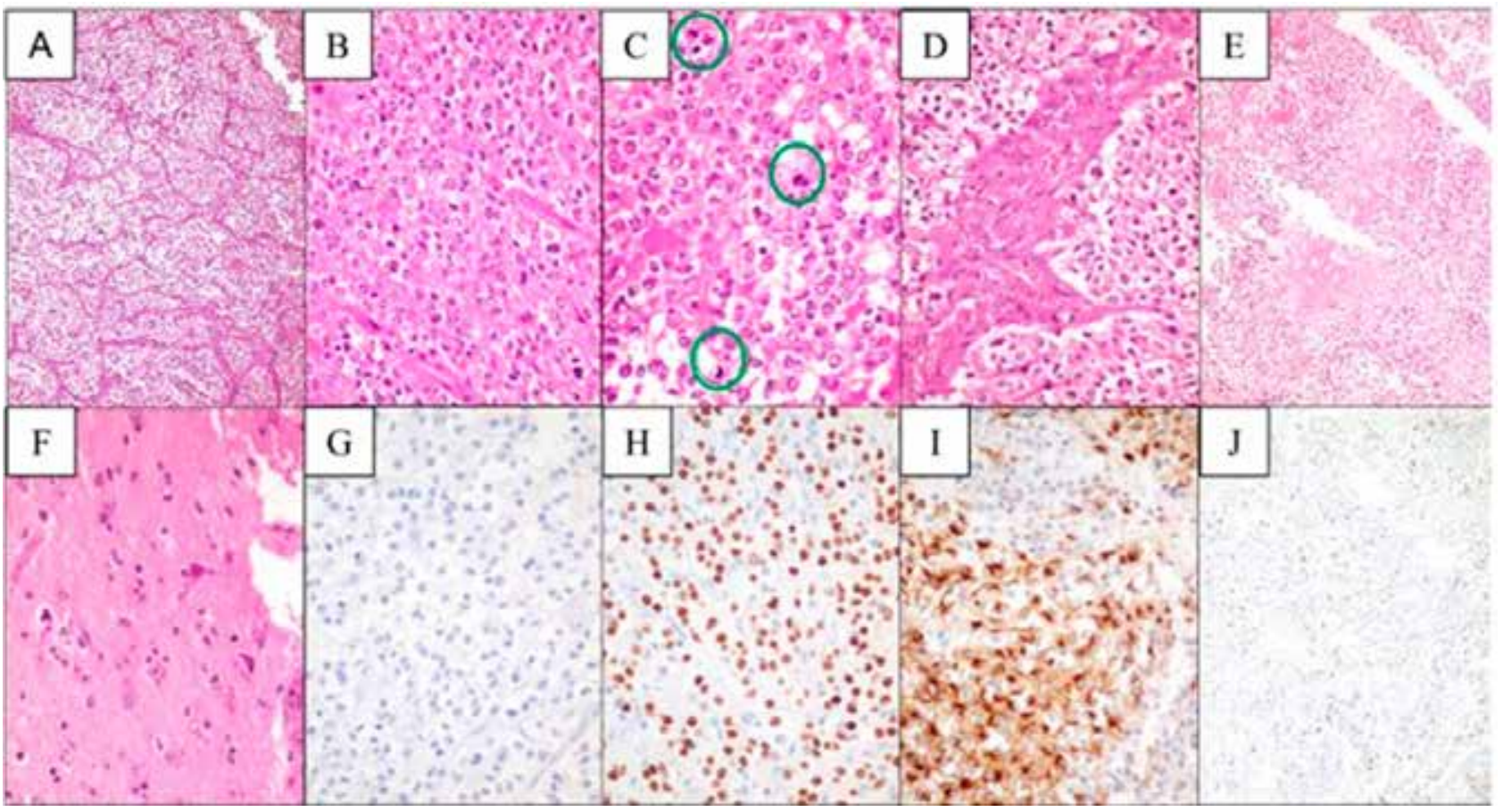

Figura 3: Oligodendroglioma anaplásico NOS. A: HE (100X), neoplasia con patrón de crecimiento sólido con rica red capilar intermedia. B: HE (400X), detalle celular, células de tamaño mediano, con retracción pericelular con aspecto en "huevo frito". C: HE (400X), tres figuras de mitosis en un campo de seco fuerte (círculos). D: HE (400X), hiperplasia endotelial capilar. E: HE (100X), necrosis central. F: HE (400X), estructuras secundarias de Scherer en la corteza adyacente. G: Inmunohistoquímica Neurofilamentos (400X), negativo en células neoplásicas. H: Inmunohistoquímica Olig2 (400X), positivo nuclear en células neoplásicas. I: Inmunohistoquímica PAGF (400X), positivo citoplásmico en células neoplásicas. J: Inmunohistoquímica Ki67 (100X), positivo nuclear en el 20\% de las células neoplásicas.

empalizada similar a la del glioblastoma. La aparición de necrosis destaca una proliferación significativa típica de los tumores de alto grado y del intento de compensar esta hipoxia mediante la aparición de proliferación microvascular(6).

En nuestro caso, la RM mostró una gran lesión de aspecto quístico en ubicación cortico-subcortical fronto parietal con depósitos de hemosiderina en la superficie caudal, formando un nivel líquido-líquido lo que indican sangrado intralesional.

\section{Referencias}

1. Koeller KK, Rushing EJ. From the archives of the AFIP. Oligodendroglioma and its variants: Radiologic-pathologic correlation. Radiographics. 2005; 25(6): 1669-1688.

2. Ankenbrandt W, Paleologos N. Imaging of Oligodendrogliomas. Handb Neuro-Oncology Neuroimaging Second Ed. 2016; (December 2015): 461-469.

3. International Agency for Research on Cancer. WHO Classification of Tumours of the Central Nervous System
WHO series on histological and genetic typing of human tumours. Editores Webster K. Cavenee, David N. Louis, Hiroko Ohgaki, Otmar D. Wiestler. Editor International Agency for Research on Cancer, 2007. ISBN 9283224302, 9789283224303: 309 páginas.

4. Figini M, Riva M, Graham M, Castelli GM, Fernandes $B$, Grimaldi M, et al. Prediction of isocitrate dehydrogenase genotype in brain gliomas with MRI: Single-shell versus multishell diffusion models. Radiology. 2018; 289(3): 788-796.

5. Hu N, Richards R, Jensen R. Role of chromosomal $1 p / 19 q$ co-deletion on the prognosis of oligodendrogliomas: A systematic review and meta-analysis. Interdiscip Neurosurg Adv Tech Case Manag. 2016; 5: 58-63. Available from: http://dx.doi.org/10.1016/j. inat.2016.06.008

6. Kaufmann TJ, Giannini C, Guerin JB, Eckel LJ, Morris JM, Johnson DR. Updates to the WHO Brain Tumor Classification System: What the Radiologist Needs to Know. RadioGraphics. 2017; 2164-2180.

7. Lanman TA, Compton JN, Carroll KT, Hirshman BR, 
Ali MA, Lochte B, et al. Survival patterns of oligoastrocytoma patients: A surveillance, epidemiology and end results (SEER) based analysis. Interdiscip Neurosurg Adv Tech Case Manag. 2018; 11: 70-75. Available from: https://doi.org/10.1016/j.inat.2017.07.017

8. Khalid L, Carone M, Dumrongpisutikul N, Intrapiromkul J, Bonekamp D, Barker PB, et al. Imaging characte- ristics of oligodendrogliomas that predict grade. Am J Neuroradiol. 2012; 33(5): 852-857.

9. Karlberg A, Berntsen EM, Johansen $H$, Myrthue $M$, Skjulsvik AJ, Reinertsen I, et al. Multimodal 18F-Fluciclovine PET/MRI and Ultrasound-Guided Neurosurgery of an Anaplastic Oligodendroglioma. World Neurosurg. 2017; 108: 989.e1-989.e8. 